\title{
Системы тестирования на ЭМС микросхем и печатных плат
}

\author{
Д. Кондрашов ${ }^{1}$, А. Шостак ${ }^{2}$
}

УДК 621.317 | ВАК 05.11 .01

\begin{abstract}
Отказ прибора при прохождении испытания на электромагнитную совместимость (ЭМС) обходится предприятиям дорого как с финансовой, так и с временной точек зрения. Одна из причин такой ситуации - неустойчивость микросхем, печатных плат и других электронных компонентов, входящих в состав готовых изделий, к воздействиям помех. Предотвратить либо уменьшить возможные отказы еще на этапе разработки поможет всестороннее углубленное тестирование на ЭМС отдельных компонентов изделий.
\end{abstract}

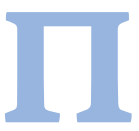

роводя сертификационные испытания, можно установить, проходит или не проходит устройство испытания по стандартам на ЭМС. Если не проходит, то найти конкретную причину возникновения неисправности сложно.

Самый простой способ защиты от электромагнитных помех - экранирование изделия, однако данный способ довольно затратен и не всегда применим, так как помехи имеют различную природу и могут возникать в кабелях и цепях питания, иметь индуктивный или емкостной характер. В такой ситуации основная задача разработчика аппаратуры - найти источник излучения (помехи), приводящего к функциональной неисправности.

На рис. 1" представлено типовое распределение и взаимное влияние электрических, магнитных полей и токов в объеме законченного устройства.

При такой ситуации выявление источника помехи довольно затруднительно, так как необходима поочередная проверка каждого компонента, входящего в состав данного устройства. Используя дифференцированный подход, подразумевающий предварительное тестирование отдельных компонентов, можно упростить и ускорить процесс разработки и сертификации устройства.

Рассматривая стандарты для испытания на ЭМС готового изделия, можно заметить, что их наберется несколько десятков, но они неприменимы к платам и микросхемам, так как эти компоненты являются относительно новым объектом ЭМС-стандартизации и требуют введения особых стандартов и нормативов, относящихся исключительно к ним. В настоящее время Международной

ООО «Остек-Электро», начальник группы волновых процессов, Kondrashov.D@ostec-group.ru.

ООО "Остек-Электро", технический специалист,

Shostak.A@ostec-group.ru.

Все рисунки предоставлены компанией Langer EMV-Technik

(www.langer-emv.de) электротехнической комиссией (МЭК; англ. International Electrotechnical Commission, IEC) разработаны две группы нормативов (рис. 2), стандартизирующих методики измерения излучаемых помех (стандарт IЕC 61967) и помехоустойчивости (стандарт IEC 62132) микросхем.

Однако специалисты немецкой фирмы Langer EMV-Technik, помимо аппаратуры для испытаний микросхем по указанным стандартам, разработали аппаратуру для проведения предварительных испытаний, цель которых - определение и устранение проблемы ЭМС в микросхемах и печатных платах. В этом случае испытания микросхем проводятся с использованием оборудования и методик, соответствующих стандартам для тестирования на ЭМС готовых изделий. Такие испытания позволяют выявлять источники помех и неустойчивые к воздействию помех места. Устранив данные недоработки, можно будет исключить схемотехнический "просчет» при непрохождении готовыми изделиями испытаний на ЭМс.

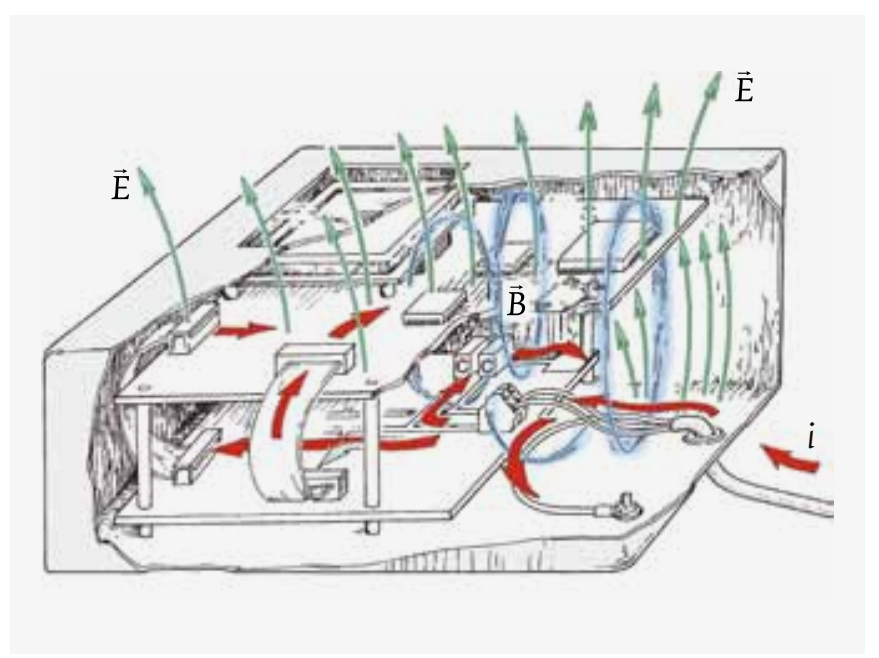

Рис. 1. Распределение электрических, магнитных полей и токов в корпусе готового устройства 


\section{Помехоэмиссия}

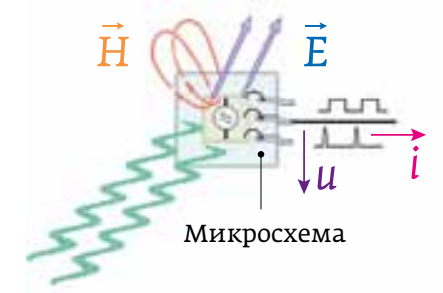

Кондуктивные помехи

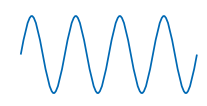

BY

(IEC 61967-4)

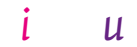

P600 P700

\begin{abstract}
Емкостные / индуктивные помехи
\end{abstract}

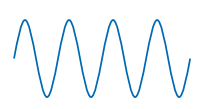

Bप

(IEC 61967-2, IEC 61967-3)

P1600, P1700, ICR-H ICR-E
$\overrightarrow{\mathrm{H}} \quad \overrightarrow{\mathrm{E}}$

\section{Помехоустойчивость}

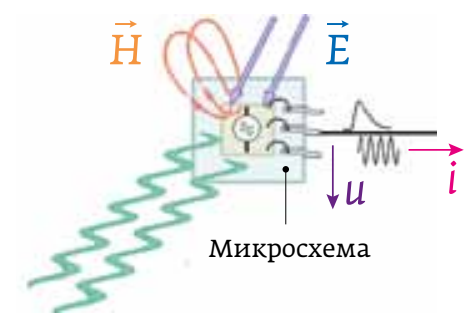

Кондуктивные помехи

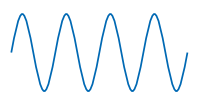

Bप (IEC 62132-4)

i, u P500

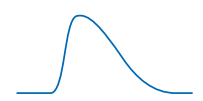

Импульсы (IEC 62215-3)

i u

P200 P300

Емкостные / индуктивные помехи

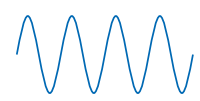

Bप

(IEC 62132-2)

$\overrightarrow{\mathrm{H}} \quad \overrightarrow{\mathrm{E}}$

P1400 P1500

Рис. 2. Группы ЭМС-стандартов для печатных плат и микросхем с решениями, предложенными компанией Langer EMV-Technik для каждого из стандартов. Рхxх и Рхxхx - модели пробников

Для примера рассмотрим испытания по стандарту на устойчивостьк электростатическому разряду в соответствии с ГОСТ 30804.4.2-2013 (IЕC 61000-4-2:2008). Он не является стандартом помехоустойчивости для печатных плат и микросхем. Однако при воздействии высоковольтного электростатического разряда можно определить, выдерживает ли микросхема данное воздействие, не нарушается ли выполнение заложенных
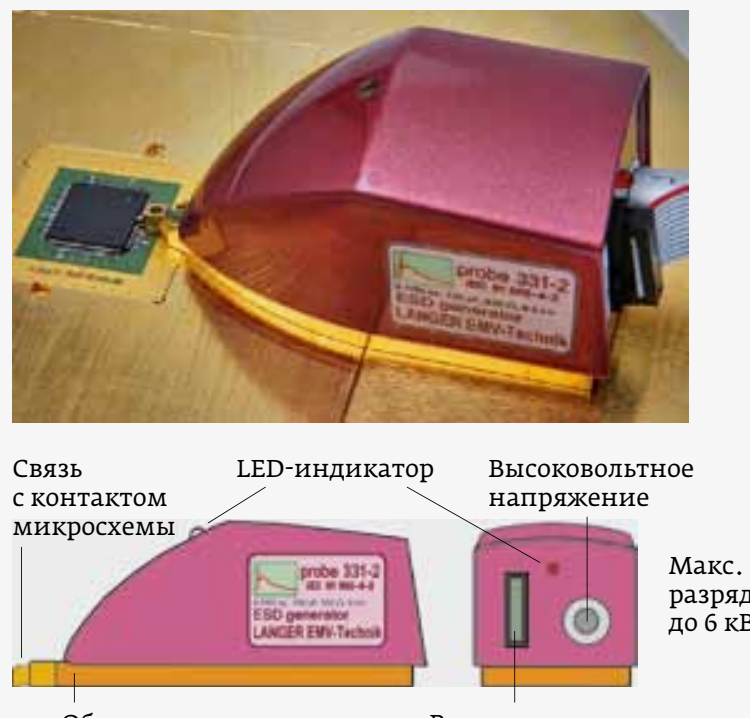

a)

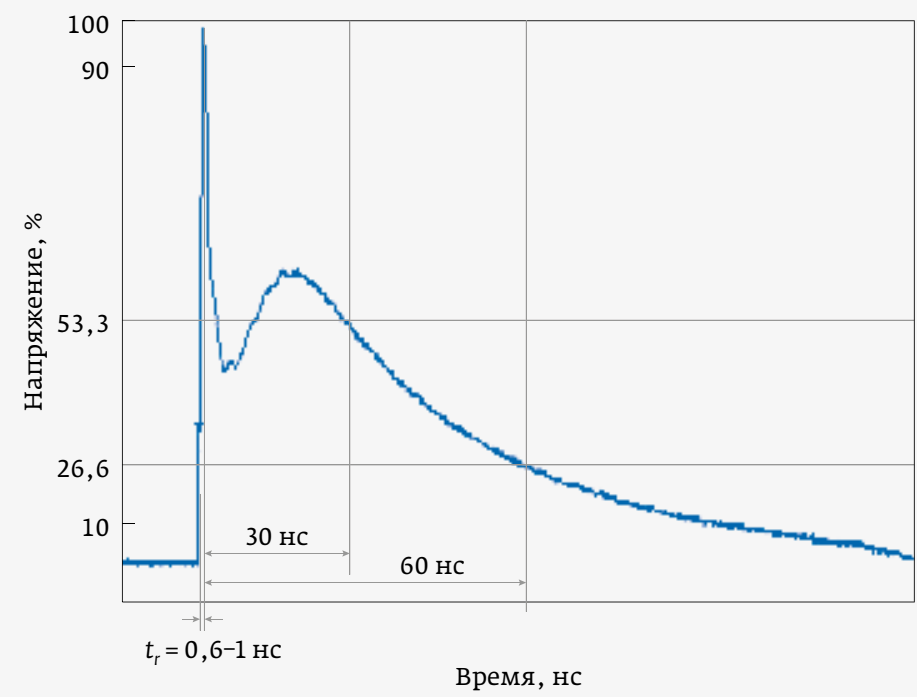

б)

Рис. 3. Устройство ввода электростатического разряда: а - внешний вид; 6 - форма импульса разряда (в соответствии с ГОСТ 30804.4.2-2013). $t_{\mathrm{r}}$ - время нарастания импульса 


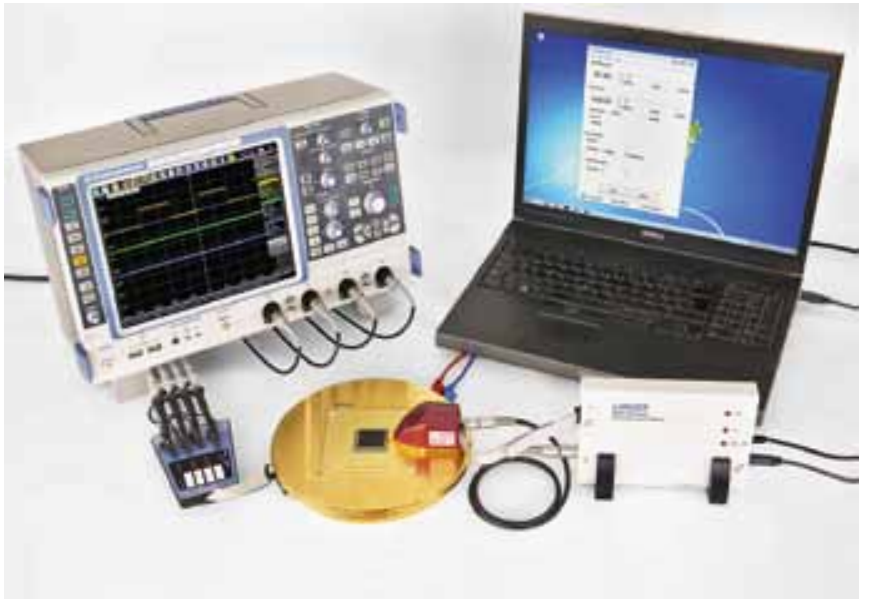

Рис. 4. Установка для проведения испытаний на устойчивость к высоковольтному электростатическому разряду

программ, не проявляются ли на ней механические повреждения.

Используя специальное устройство ввода (рис. 3) и установку, в состав которой оно входит (рис. 4), можно воздействовать разрядом на каждую ножку микросхемы. Это помогает выявить причину неисправности, определив тип паразитной связи (емкостная, индуктивная, кондуктивная) и параметры помехи (время нарастания, форма сигнала, ток, напряжение, напряженности электрического или магнитного полей и др.), влияющие на микросхему. Данная информация позволяет разрабатывать меры по повышению помехозащищенности.

Удобным инструментом для проверки на помехоустойчивость печатных плат и микросхем являются также пробники для ввода (инжекции) помехи (рис. 5). Инжекционные пробники компании Langer EMV-Technik можно разделить на портативные (ручные) и пробники ICl. Их используют как источник паразитного электромагнитного поля (помехи). Благодаря своим малым габаритам, они позволяют вводить помеху в небольшие области на плате: контактные дорожки, ножки микросхем, цепи связи и питания.

Портативные пробники (рис. 6) являются пассивными и подключаются к генератору наносекундных или микросекундных помех в соответствии со стандартами ГОСТ 30804.4.4-2013

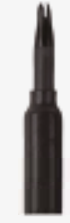

Портативные пробники:

- используются с генератором наносекундных импульсных помех;

- средняя разрешающая способность;

- ввод помех в цепи питания печатных плат (Power Glitch on Blocking capacitors)

\section{Инжекционные пробники}

ICI-пробники:

- высокая разрешающая способность;

- встроенный генератор

импульсных помех;

- ввод помех

в кристалл

(Open-Die Injection)
Рис. 5. Пробники компании Langer EMV-Technik для ввода помех в микросхемы и платы

(IEC 61000-4-4 : 2004), ГОСТ IEC 61000-4-5-2017. Портативные пробники имеют множество моделей, выбор которых зависит от типа вводимого поля, размеров исследуемой области и диапазона частот. Пробники ICI - активные. Они имеют собственный генератор импульсов и используются для ввода помехи в интегральные схемы. Благодаря высокому разрешению можно воздействовать на отдельные области на микросхеме.

Микропробник ICI подключается к автоматической системе сканирования ICS 105 (рис. 7), в которой управление

\section{Магнитное поле}
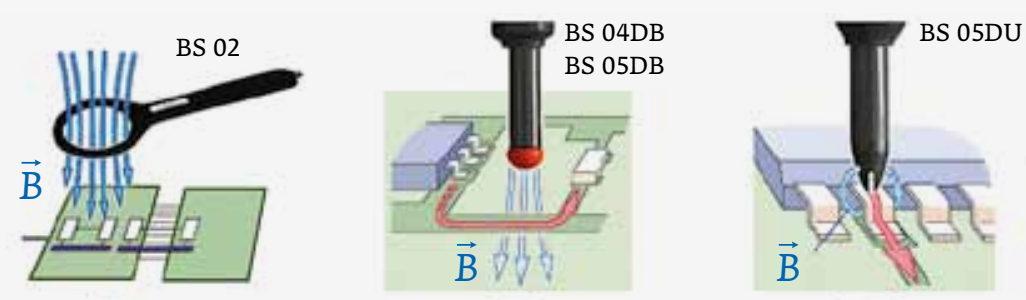

Электрическое поле
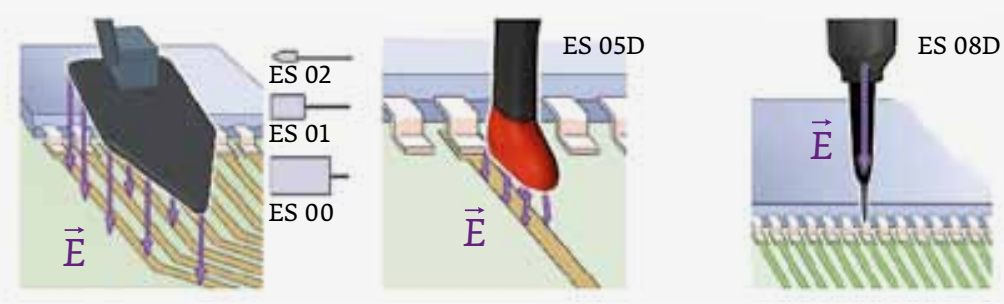

Рис. 6. Портативные инжекционные пробники компании Langer EMV-Technik 


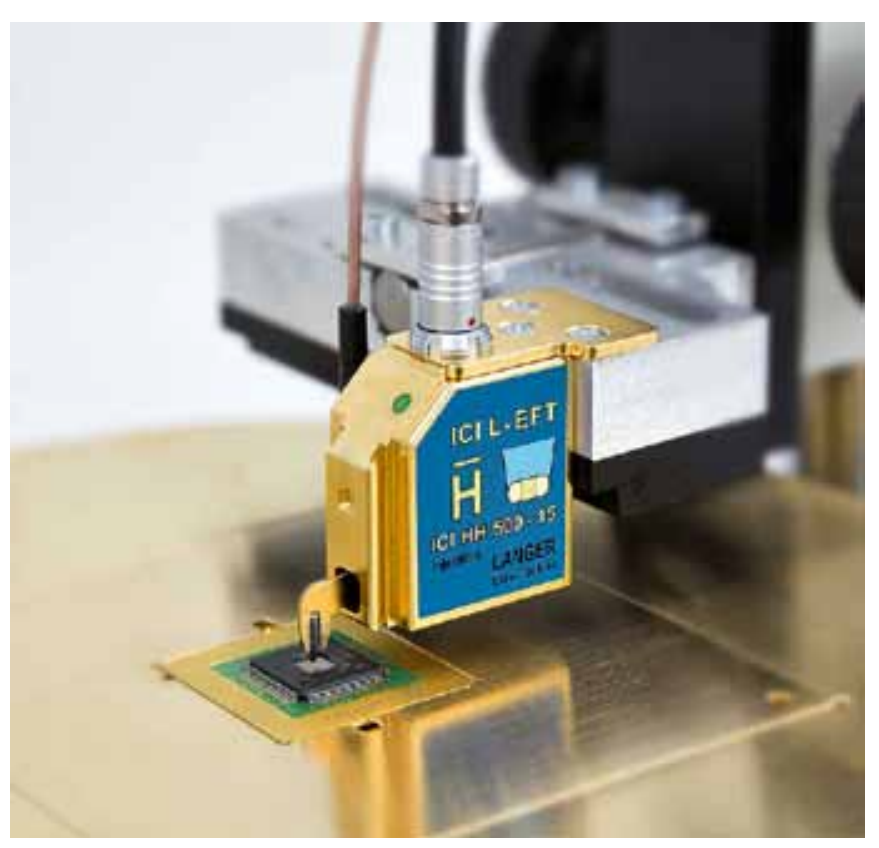

Рис. 7. Установка для ввода помех в микросхемы

измерениями проводится с помощью ПК с предустановленным программным обеспечением (ПО). Существуют пробники магнитного/электрического поля. Они генерируют мощные (несколько киловольт) импульсы локализованного поля со временем нарастания 2 нс, контролируемые с помощью осциллографа. Есть третий вид пробников - ВBI (Body Bias Injection) Probe. Они действуют аналогично генератору электростатического разряда и используются для контактного ввода импульсов тока в подложку интегральной схемы.

Помимо испытаний на помехоустойчивость, не менее важным является измерение собственного излучения (помехоэмиссии) печатных плат и микросхем. Для возникновения излучения необходимы источник ВЧ-сигнала и антенна. Источником Вч-сигнала может стать сама плата и ее компоненты, антенной - контактные дорожки, кабели и корпус устройства. При этом устройство излучает в ближней зоне. Для измерений излучения такого рода необходимо использовать специальные пробники ближнего поля (рис. 8). Утаких пробников также есть много моделей, рассчитанных на различные типы поля, размеры исследуемой области и диапазоны частот.

Для корректного измерения собственного излучения необходимо

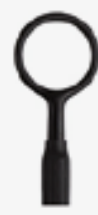

Портативные

- средняя разрешающая способность;

- измерения в ВЧ-диапазоне;

- измерение излучения плат (Board level)

Возможное применение:

- антенны для NFC;

- контроль энергопотребления (Power consumption) (ручные):

\section{Микропробники}

ICR:

- высокая разрешающая способность;

- измерения в ВЧ-диапазоне;

- измерение излучения интегральных схем (1C level)

Возможное применение:

- определение излучающих областей кристалла схемы;

- сканирование кристалла схемы (Open-Die Scan)
Рис. 8. Пробники компании Langer EMV-Technik для измерения собственного излучения плат и микросхем

пространство, в котором будет происходить ослабление внешних электромагнитных полей. Такое пространство могут обеспечить экранированные камера или бокс. В линейке Langer EMV-Technik есть складная экранированная камера (рис. 9), представляющая собой металлический каркас и специальную металлосодержащую ткань, препятствующую проникновению электромагнитных волн. Поместив в такую камеру тестируемую плату и используя портативный пробник, подключенный канализатору спектра, можно измерять собственное излучение платы.

Далее рассмотрим микропробники ближнего поля ICR. Они работают вместе с автоматическими системами

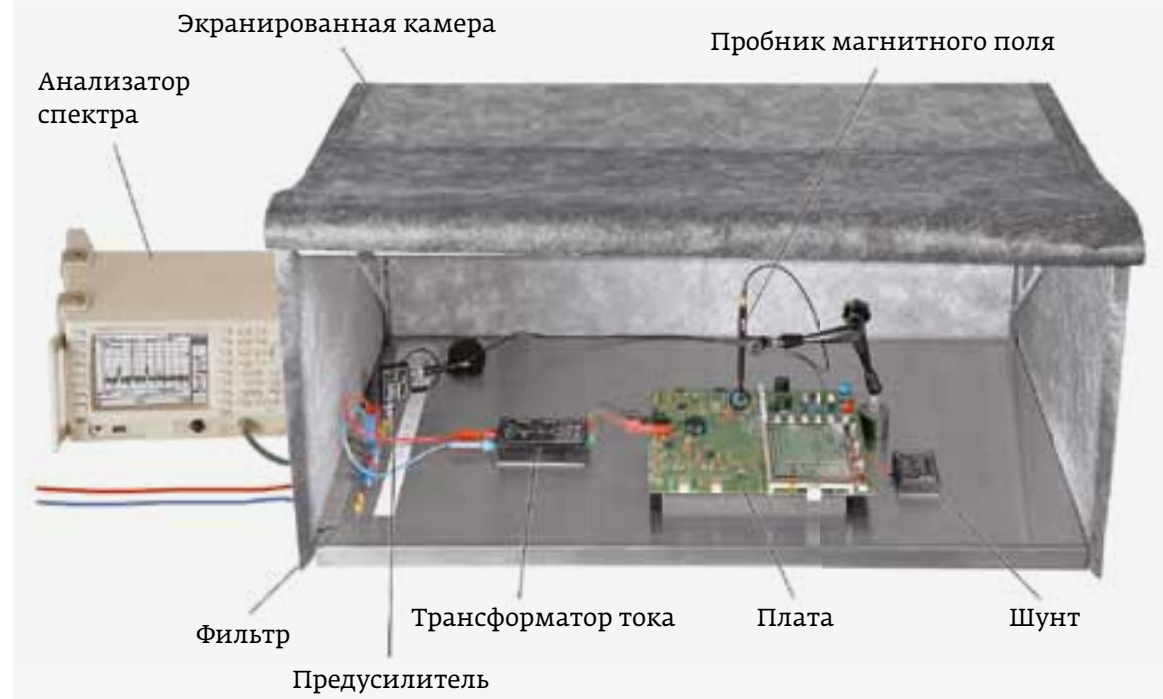

Рис. 9. Установка для измерения собственного излучения печатных плат с помощью портативных пробников 


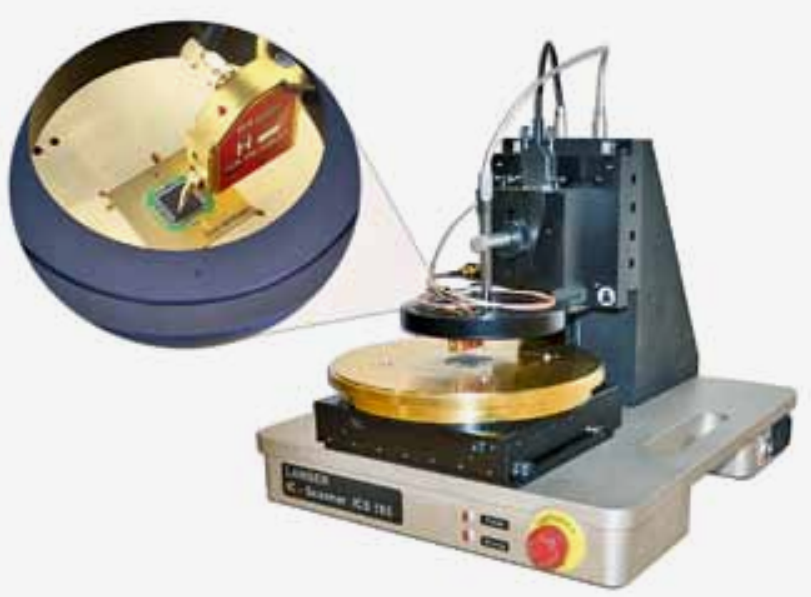

Рис. 10. Установка для измерения собственного излучения печатных плат с помощью микропробников ICR

сканирования ICS 105. Частотный диапазон измерения микропробников - от 200 кГц до 6 ГГц. От портативных их отличает следующее: во-первых, наконечник датчика намного меньше. Диаметр наконечника может достигать 100 мкм, что позволяет измерять эмиссию микросхем. Во-вторых, датчики имеют встроенный предусилитель, который питается от отдельного источника. Конструкция пробников такова, что пробник, измеряющий магнитную компоненту поля, экранирован от электрического поля, и, наоборот, пробник, измеряющий электрическую компоненту поля, экранирован от магнитного поля. Данное конструктивное решение позволяет измерять исключительно один тип поля.

Прежде чем использовать данные микропробники, микросхему необходимо разместить на специальной подложке с оснасткой, которая изготавливается для каждого типа схемы индивидуально. С помощью данной подложки можно управлять различными режимами работы микросхемы, регулируя уровень собственного излучения.

Микропробник ICR, аналогично микропробнику ICI, подключается к автоматической системе сканирования ICS 105 (рис. 10). Микропробник передает данные на анализатор спектра с шагом измерения 10 мкм, определяя наибольшее излучение на всей площади микросхемы и перемещаясь непосредственно в место этого излучения. На основе полученных данных ПО строит трехмерное изображение (рис. 11) распределения излучения на микросхеме. Наиболее мощное излучение отображается красным цветом. Также с помощью ПО можно измерять поле у каждой ножки (вывода) микросхемы.

Используя аппаратуру Langer EMV-Technik, можно решать различные задачи по проверке помехоустойчивости
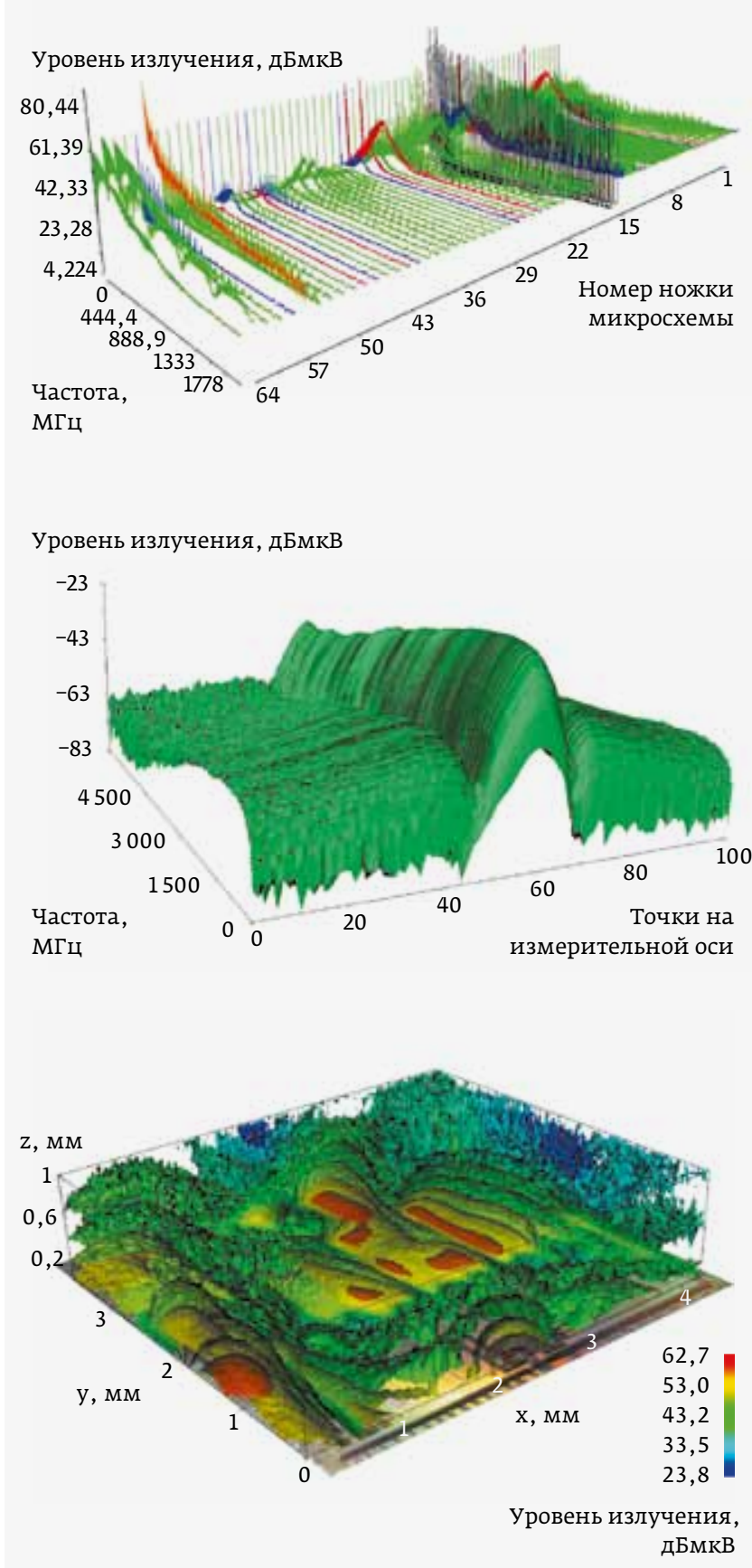

Рис. 11. Результаты измерений плат и микросхем, полученные с помощью ПО компании Langer EMV-Technik

и излучения печатных плат и интегральных микросхем, увеличивая гарантию прохождения сертификационных испытаний на ЭМс законченными изделиями.

Подробнее с решениями для испытаний на ЭМС компании Langer EMV-Technik можно ознакомиться на сайте www.ostec-electro.ru или отправив запрос по электронной почте ostecelectro@ostec-group.ru. 


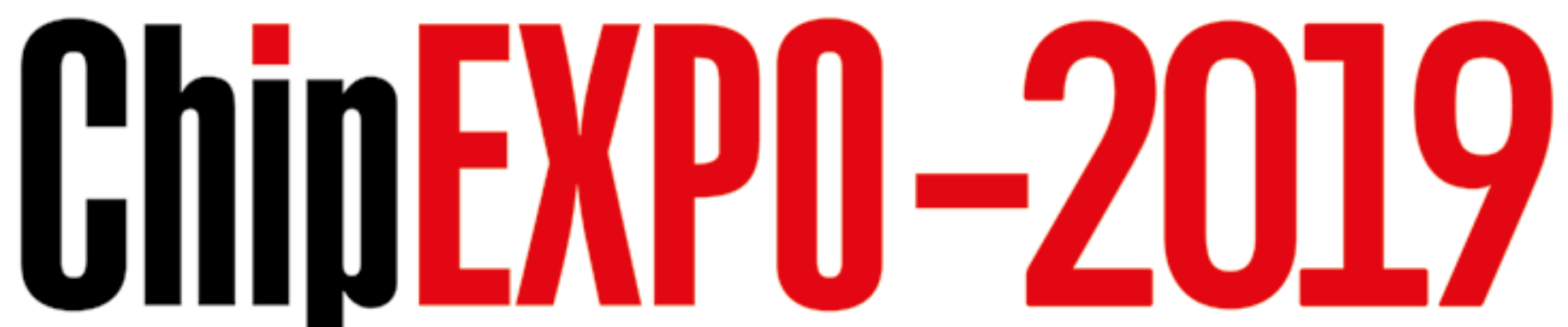

\section{КОМПОНЕНТЫ | ОБОРУДОВАНИЕ | ТЕХНОЛОГИИ}
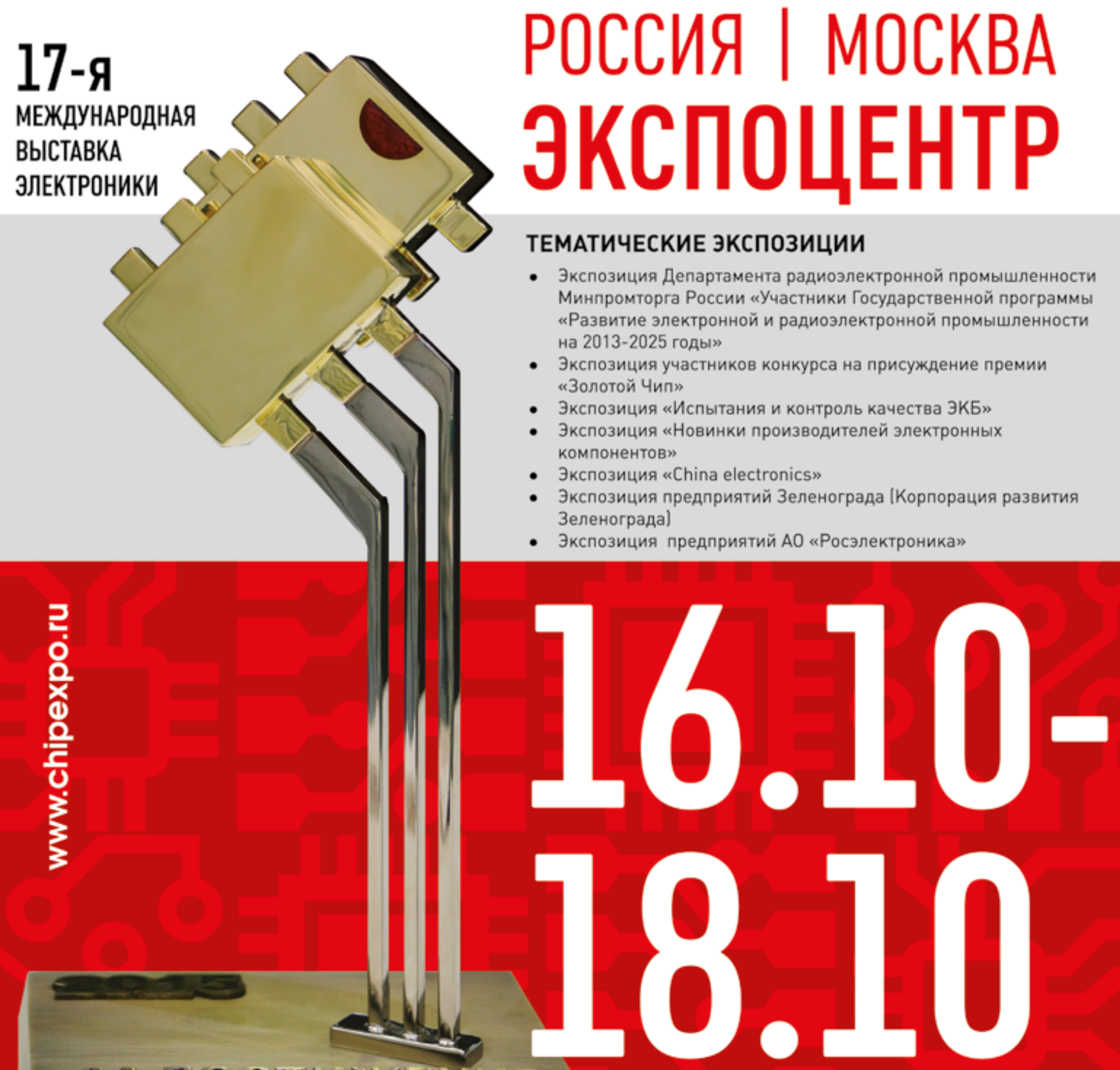

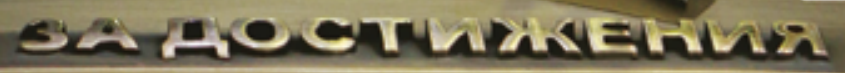

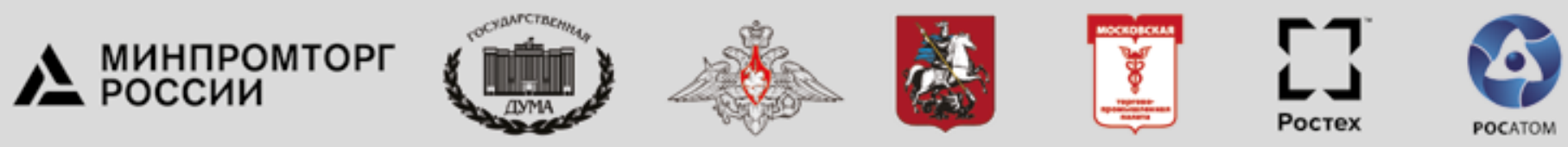

